\title{
Cryptorchidism: a registry based study in Sweden on some factors of possible aetiological importance
}

\author{
MATS HJERTKVIST, ${ }^{1}$ JAN-ERIK DAMBER, ${ }^{2}$ AND ANDERS BERGH ${ }^{3}$ \\ From the Departments of ${ }^{1}$ Surgery, ${ }^{2}$ Urology and Andrology, and ${ }^{3}$ Pathology, University of Umeå, Umeå, Sweden.
}

\section{ABSTRACT}

Study objective: To examine the epidemiological characteristics of boys with cryptorchidism.

Design: Cohort survey using the Swedish personal identification code and Medical Birth Registry to link cryptorchid boys with their pregnancy and delivery data. They were compared with the total population of male births during the same period.

Subjects: 2861 cryptorchid boys born in Sweden between 1973 and 1982 were identified. Of these 437 could not be linked with their Medical Birth Registry information for various reasons, or were excluded because they were under 1 year of age at the end of 1982, leaving 2424 cases for study. The reference population comprised all boys born in Sweden during the same period $(n=458601)$.

Measurements and main results: For each study variable, the observed number of cases among boys with cryptorchidism was compared with the expected number calculated from the whole population. A significant increase in intensity ratio for cryptorchidism was found with the first birth, caesarean section, toxaemia of pregnancy, and certain congenital abnormalities (hypospadias and subluxation of the hip). It was also more common in small for dates infants. There was a seasonal variation in cryptorchidism, with increased incidence in January-March.

Conclusions: Cryptorchidism may be caused by hormonal influences during pregnancy, which could be affected by utero-placental factors involving placental dysfunction or by daylight hours, through pineal activity.

Cryptorchidsm is one of the most common congenital malformations in males. There are reports ${ }^{12}$ showing an increase in incidence. The close association between cryptorchidism and testicular malignancy ${ }^{3-5}$ and infertility ${ }^{6}$ makes the condition clinically important. However, the aetiology of this disorder is little understood. Testicular descent is believed to take place in two phases. ${ }^{7}$ During the first phase, occurring during the first trimester, the testes descend from their intra-abdominal position to the groin and this phase is believed to be independent of androgens. The second phase occurs during the third trimester, when the testes descend to the scrotal sac. It is believed that this second phase of descent is androgen dependent. ${ }^{7}$ Theoretically, any condition in the mother or in the fetus causing disturbed androgen production may lead to cryptorchidism, although such conditions are hard to define. Previous studies have suggested some risk factors which might be associated with the aetiology of cryptorchidism, such as impairment of the placental function, ${ }^{8}$ abnormal chorionic gonadotrophin (hCG) secretion, ${ }^{8}$ exposure to oestrogens, ${ }^{8}$ and breech presentation in labour. ${ }^{9}$ These factors may cause an impairment of testicular androgen production in the fetus. The present study was designed to investigate characteristics of the mother and/or the child which are possibly related to cryptorchidism and are perhaps aetiological factors for this malformation. This was done by use of a nationwide public health registry.

\section{Methods}

Since 1973, a Medical Birth Registry in Sweden has collected medical information on all births. It is based on a standardised record form used in the whole country (with a local variant in one county) for medical care in maternity health centres (which nearly every pregnant woman attends), delivery units (practically all deliveries occur in hospitals), and at examination of the newborn infant (nearly all infants are examined by a qualified paediatrician). The form is 
used for information exchange between maternity health centres, delivery units and child health centres. A copy of the form goes to the National Board of Health and Social Welfare and forms the basis for the registry. It contains among other things some social data, data on the pregnancy and the newborn infant, and diagnoses referring to pregnancy, delivery and the newborn. Such diagnoses are submitted in a coded form, using the Swedish modification of the ICD8 classification. ${ }^{10}$

Another registry, The Hospital Discharge Registry, contains information on all patients discharged from hospitals, including discharge diagnoses (coded as ICD8) and operation codes. This registry does not cover the whole country, as only approximately $70 \%$ of the counties participate.

In both registries, each individual is identified by the unique personal identification code which every Swedish citizen gets soon after birth. This makes it possible to link the two registries to each other.

All boys over the age of one year in the Hospital Discharge Registry with the diagnosis of cryptorchidism and/or who had received an orchidopexy were identified. During the period studied (1973-1982) nearly all boys with this condition were treated surgically. A total of 2861 cases were identified. By linkage to the Medical Birth Registry (1973-1981), information concerning pregnancy, delivery and the conditions in the neonatal period were linked to each case. No linkage was possible in 437 cases, either because their identification numbers were incomplete or wrong in either file, or because they were not born in Sweden but had immigrated. In these cases it was not possible to compare variables with the group in which linkage was achieved. Infants born in 1982 were excluded because this cohort had not yet reached the age of 1 year at the end of 1982. The diagnosis is not accepted under 1 year of age because of the known possibility of spontaneous testicular descent during the first year of life. This left 2424 cases for further study.

As a comparison group, all 458601 boys born in 1973-1981 and registered in the Medical Birth Registry were used. The table presents the annual number of cases and total male births in each year.

\section{Statistical methods}

For each study variable, the observed number of cases among the boys with cryptorchidism was compared to the expected number, calculated from the total group. This comparison was often made after stratification for different variables, eg, year of birth, maternal age and/or parity, delivery unit. The ratio between the observed and expected number is called intensity ratio, a neutral term which does not imply causality. The
Table The number of cases of cryptorchidism born 1973-81 and operated on at 1 year of age or older 1974-82 and all males born per year 1973-81.

\begin{tabular}{lll}
\hline $\begin{array}{l}\text { Year of } \\
\text { Birth }\end{array}$ & Cases & $\begin{array}{l}\text { All males } \\
\text { born }\end{array}$ \\
\hline 1973 & 594 & 55876 \\
1974 & 521 & 56269 \\
1975 & 441 & 52860 \\
1976 & 303 & 50250 \\
1977 & 240 & 49274 \\
1978 & 167 & 47580 \\
1979 & 86 & 49029 \\
1980 & 46 & 49718 \\
1981 & 26 & 47745 \\
\hline
\end{tabular}

statistical significance of an intensity ratio was evaluated with $\chi^{2}$ statistics when the expected number exceeded 5 , and the approximate $95 \%$ confidence interval (CI) was determined with Miettinen's approximation. ${ }^{11}$ When the expected number was 5 or less, a Poisson model was applied instead. Seasonality was studied with the use of Edward's model ${ }^{12}$ where the expected number of cases each calender month was adjusted for variations in total number of births that year and month.

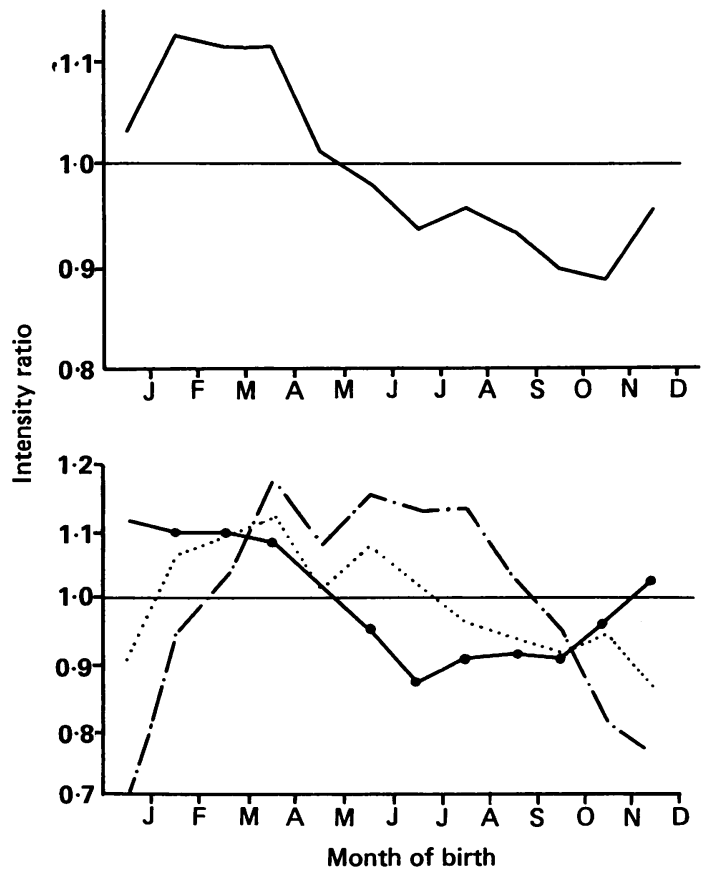

Fig 1 Intensity ratios (IR) for cryptorchidism in different months of the year. The upper panel shows the curve for the whole of Sweden and the lower for different geographic regions (south, middle and north). 


\section{Results}

SEASONALITY:

A seasonal variation in the intensity ratio of birth of boys with cryptorchidism was found. There was a maximum intensity ratio from January to March and a minimum in November. Figure 2 shows the intensity ratio for cryptorchidism in the whole of Sweden and in different geographical areas, after adjustment for the seasonal variations in the number of births. Edward's formula ${ }^{12}$ gives $\chi^{2}=25.9$ at $2 \mathrm{df}$ for the total material, $\mathrm{p}<0.001$. For each one of the three areas, a corresponding calculation gives: south, $\chi^{2}=11.0$ $(\mathrm{p}<0.01)$; middle, $\chi^{2}=4.5(\mathrm{p}>0.05)$; and north, $\chi^{2}=6.2(\mathrm{p}<0.05)$. The seasonality in the middle area thus does not reach statistical significance, but all three areas may show random variation from the same seasonality.

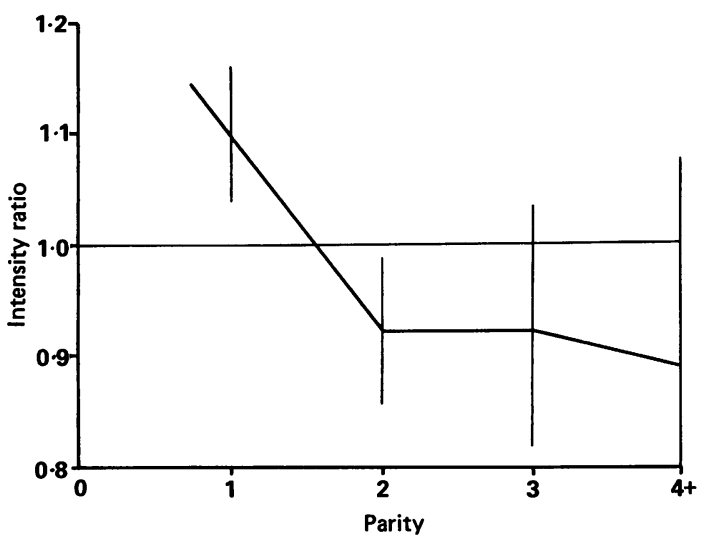

Fig 2 Intensity ratios (IR) for cryptorchidism in relation to parity (with $95 \%$ confidence intervals).

CAESAREAN SECTION:

Among the cryptorchid boys, 264 (10.9\%) were delivered by caesarean section. The expected number, after standardisation for year of birth and delivery unit, was $197 \cdot 6$, intensity ratio $=1 \cdot 33, \mathrm{p}<0.001,95 \%$ $\mathrm{CI}=1 \cdot 18-1 \cdot 51$. The expected number after standardisation for birthweight and year of birth was $202 \cdot 6$, intensity ratio $=1 \cdot 30, \mathrm{p}<0 \cdot 001,95 \% \mathrm{CI}=1 \cdot 15$ $1 \cdot 47$.

\section{MATERNAL FACTORS}

Parity-Figure 2 shows the intensity ratio of cryptorchidism in relation to parity after adjustment for year of birth, birth weight and age of the mother. It was found that there was an increased intensity ratio for cryptorchidism in the first born child and that there was no difference in subsequent births. The intensity ratio for first births $v$ higher order births was $1 \cdot 11$, $\mathrm{p}<0.001$, with a $95 \%$ CI of $1.05-1 \cdot 18$.

Maternal age - When adjusted for parity, year of birth and birthweight there was no significant effect of maternal age on the intensity ratio for cryptorchidism, as seen in fig 3 . When calculated without adjustment there was an increased intensity ratio of cryptorchidism in cases born to younger mothers but this was secondary to the effect of parity.

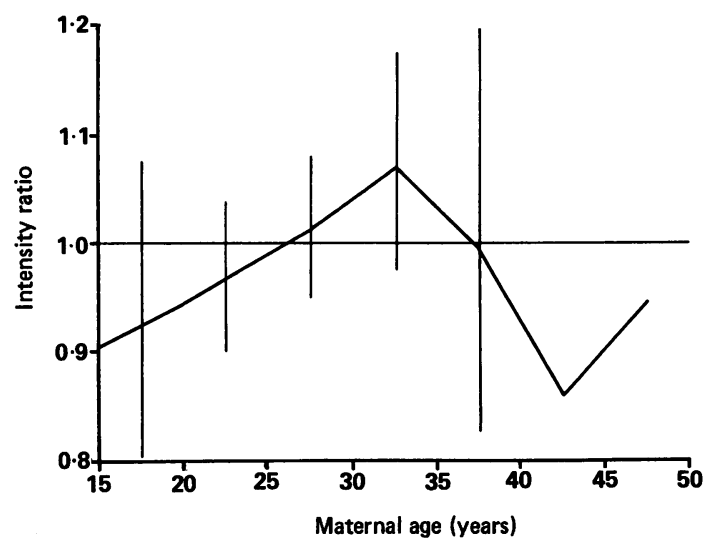

Fig 3 The intensity ratios (IR) for cryptorchidism in relation to maternal age (with $95 \%$ confidence intervals).

Maternal illness-In 14 cases the mother was found to have diabetes mellitus. When compared to the expected number $(8 \cdot 14)$, this gives an intensity ratio of $1.7(p<0.05)$. Pregnancy toxaemia occurred in 275 mothers. The expected number when adjusted for

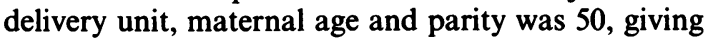
an intensity ratio of $5.4 \quad(\mathrm{p}<0.001)$. Pregnancy hyperemesis occurred in 12 cases, the expected number being $8 \cdot 8$. This gives an IR of $1 \cdot 4$, which was not statistically significant.

Length of gestation-Figure 4 shows the relationship between length of gestation and cryptorchidism. Adjustment has been made for the age of the mother, parity and year of birth. Calculation of pregnancy duration is based on the date of the last menstrual period, or if not available or clearly wrong, on the gestational assessment made by the hospital. It was found that there was no significant influence of gestational age on intensity ratio for cryptorchidism diagnosed at or after one year of age.

\section{CHILD FACTORS}

Birth weight-The distribution of cryptorchidism in birth weight groups, divided into $500 \mathrm{~g}$ bands, is shown in fig 5. Adjustment was made for year of birth, age of the mother and parity. There was an intensity ratio of 


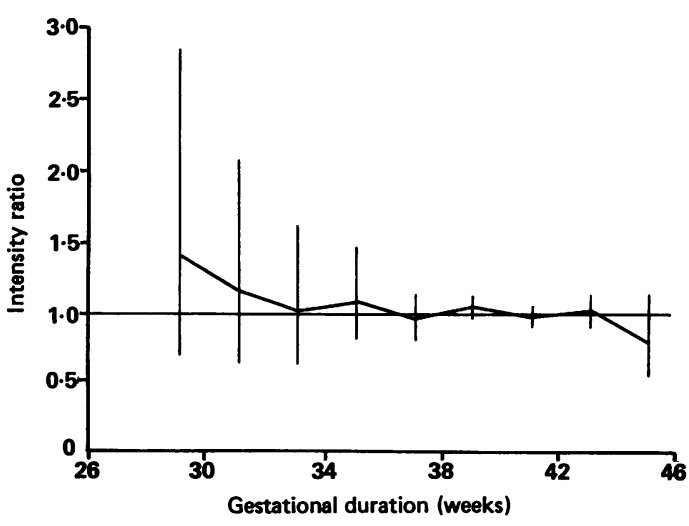

Fig 4 The intensity ratios (IR) for cryptorchidism in relation to gestational duration (with $95 \%$ confidence intervals).

$1.5(95 \% \mathrm{CI}=1.3-1.8)$ for birth weights between 1.5 and $2.5 \mathrm{~kg}(\mathrm{p}<0.001)$ but there was also a significantly increased risk for the $1.0-1.5$ and $2.5-3.0 \mathrm{~kg}$ classes.

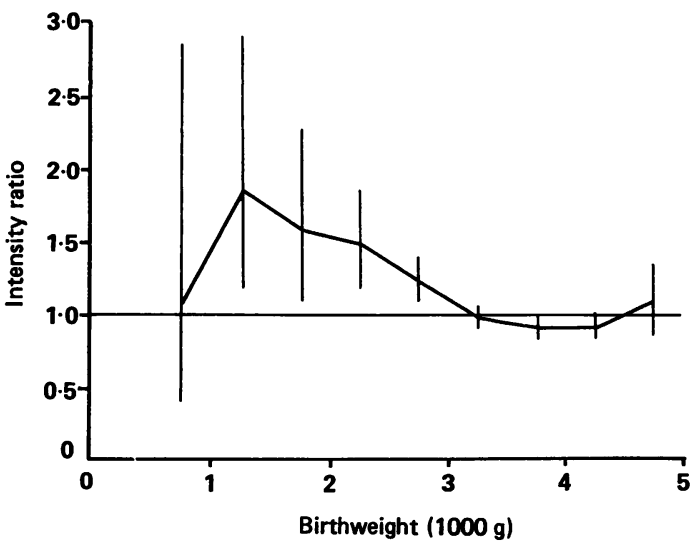

Fig 5 The intensity ratios (IR) for cryptorchidism in relation to birth weights (with $95 \%$ confidence intervals).

When birth weight and length of gestation were considered together (figs 4 and 5) there was an increased intensity ratio for cryptorchidism in boys with subnormal birth weight but not in those with subnormal gestation. This means that these boys were small for dates, irrespective of their length of gestation.

Congenital malformations - Only $30 \%$ of the cases had a diagnosis of cryptorchidism in the newborn period. This is close to the figure of $34 \%$ given in reference 1 . The distribution of congenital malformations in infants with cryptorchidism deviated from that expected from the total population with respect to only three groups: (1) The observed number of heart malformations was 16 and the expected number was 14-2. This difference may be random. (2) Hypospadias was observed in 12 cases and the expected number was 4.5 , which gives an intensity ratio of $2.7(p<0.01), 95 \%$ $\mathrm{CI}=1 \cdot 4-4 \cdot 7$. (3) Dislocation or subluxation of the hip was observed in 22 cases and the expected number, after adjustment for delivery unit and year of birth, was 8.9 which gives an intensity ratio of $2.5(<0.001)$, $95 \% \mathrm{CI}=1 \cdot 7-3 \cdot 7$.

\section{Discussion}

In this study we observed a seasonal variation in the incidence of cryptorchidism in Sweden. An increased incidence was found in boys born in March and a decreased incidence in boys born in November. In the middle area, seasonality was not statistically significant, perhaps due to small numbers. A similar seasonal variation has been reported both in Hungary and in Britain. ${ }^{1314}$ The incidence of two other diseases in the male reproductive system, hypospadias and testicular cancer, both show a similar seasonal variation. ${ }^{1516}$ The reason for this seasonal variation is unknown and difficult to explain (see Källén ${ }^{17}$ for discussion). However the sexual differentiation of the fetus, including testicular descent, is controlled by hormones, ${ }^{7}$ and seasonal differences in daylight length (via effects on the pineal gland) are known to influence ovarian hormonal activity in man. ${ }^{18}$ The seasonal variations in daylight are pronounced in Sweden, particularly in the northern part. ${ }^{18}$ Moreover, in experimental animals it has been shown that interference with pineal function in the pregnant mother influences testicular function in the male offspring. ${ }^{19}$ On the other hand our findings of a similar seasonal variation in southern and northern Sweden despite large differences in daylight hours may indicate that factors other than light could be involved. One possible example could be seasonal occurrence of certain infectious diseases impairing placental function and thus disturbing sexual development of the fetus. Impaired placental function, with general effects on fetal health and possibly reduced secretion of hCG and, as a consequence, a reduced production of testosterone and other testicular hormones in the male fetus, has been suggested as an aetiological factor for testicular maldescent. ${ }^{78}$ Some observations in this and other studies are in favour of this hypothesis:

(1) Placental insufficiency or breech labour are the most common reasons for caesarean sections in Sweden, and caesarean section was found to be more common in the cryptorchid than the control group. Breech labour as such was not associated with 
cryptorchidism and it is thus unlikely that caesarean section protects against a risk caused by breech labour as suggested by Swerdlow et al. ${ }^{9}$

(2) The intensity ratio for cryptorchidism was increased in boys born "small for date", a condition which may be due to placental insufficiency or to the fetus being unhealthy from some other cause. Earlier studies on the duration of gestation and birth weight showed an increased risk of cryptorchidism when these were subnormal. ${ }^{20}$ However, when comparing the effects of gestational length and birth weight in our study, we found that the intensity ratio for cryptorchidism was increased by low birth weight when the child was "small for date" but not when it was born preterm. Thus the mechanisms controlling testicular descent may operate normally after birth provided that the child is otherwise healthy. Up to 3 years after delivery cryptorchid boys are twice as likely as controls to have reported illnesses of some kind (other than minor colds). ${ }^{8}$ Thus some data suggest that the cryptorchid individual is somewhat unhealthy both in utero and later.

(3) Bleeding and spotting during the last trimester ${ }^{21}$ and threatened abortion ${ }^{8}$ have been reported as risk factors for cryptorchidism.

High levels of unbound oestrogens during pregnancy, as are found in toxaemia, ${ }^{320}$ have been suggested as a cause of cryptorchidism, possibly by impairing testicular hormone secretion. ${ }^{22}$ Some observations in this study support this hypothesis. (1) The study confirms that toxaemia is a risk factor for cryptorchidism (see also Källén ${ }^{23}$ ). (2) The incidence of cryptorchidism is increased in first born boys (irrespective of the age of the mother, see above) and possibly oestrogen levels may be higher in the first than in subsequent pregnancies. ${ }^{24}$ (3) Hypospadias and malformations of the hip were considerably more common in the cryptorchid than in the control group and it has been suggested that both these malformations are associated with increased oestrogen levels. ${ }^{20} 222526$. However, recently Burton et $a l^{27}$ showed that the mothers of cryptorchid boys had reduced rather than increased oestradiol levels during pregnancy, and Beard $e t a^{21}$ did not observe any increase in the incidence of cryptorchidism in boys exposed to exogenous oestrogens in utero.

Diabetes mellitus in the mother is a risk factor for cryptorchidism. The reason for this is unknown but this condition probably influences placental function and most interestingly Leydig cells are hyperplastic in newborn infants of diabetic mothers, possibly due to supranormal placental hCG secretion. ${ }^{28}$ Increased maternal hCG secretion is also observed in pregnancy toxaemia and intrauterine growth retardation, ${ }^{29}$ suggesting that the relationship between placental insufficiency and cryptorchidism is not necessarily explained by reduced but perhaps by supranormal testicular function.

In conclusion, this epidemiological study confirms several previous studies showing that diabetes mellitus, pregnancy toxaemia and caesarean section are risk factors for cryptorchidism and that the incidence of this disease is related to the month of birth. In some aspects it extends previous findings; decreased gestational length as such is not a risk factor, but a subnormal birth weight is, both at normal and subnormal gestational length. That means that these cases have retarded intrauterine growth. First born males have an increased incidence of cryptorchidism but the age of the mother is not important. The incidence of hip malformations is considerably increased among cryptorchid boys. However, it is worth remembering that only $30 \%$ of cryptorchid boys are diagnosed at birth.

The aetiology of cryptorchidism remains largely unknown but the present findings support the hypothesis that placental dysfunction causing hormonal or other disturbances in fetal health could be involved.

This work was supported by grants nos 5653 and 5935 from the Swedish Medical Research Council.

The authors are most grateful to Professor Bengt Källén, Department of Embryology, University of Lund, for valuable methodological help and suggestions. Also the assistance of Anders Ericson, National Board of Health and Welfare, and Anita Sandström, Department of Epidemiology, University of Umeå, is gratefully acknowledged.

Address for correspondence and reprints: Mats Hjertkvist, Department of Surgery, University of Umeå, S-901 87 Umeå, Sweden.

\section{References}

1 John Radcliffe Hospital Study Group. Cryptorchidism: an apparent substantial increase since 1960 . Br Med J 1986; ii: $1401-4$.

${ }^{2}$ Chilvers C, Pike MC, Forsman D, Fogelman K, Wadsworth MEJ. Apparent doubling of frequency of undescended testis in England and Wales in 1962-81. Lancet 1984; ii: 330-2.

${ }^{3}$ Henderson BE, Benton B, Jing J, Yu MC, Pike MC. Risk factors for cancer of the testis in young men. Int J Cancer 1979; 23: 598-602.

${ }^{4}$ Pottern LM, Brown LM, Hoover RN, et al. Testicular cancer risk among young men: Role of cryptorchidism and inguinal hernia. J Natl Cancer Inst 1985; 74: 377-81.

${ }^{5}$ Krabbe S, Berthelsen JG, Volsted P, et al. High incidence of undetected neoplasia in maldescended testes. Lancet 1979; i: 999-1000.

${ }^{6}$ Hadziselimovic F, Herzog B, Girard J. Cryptorchidism: Histology, fertility and treatment. Prog Reprod Biol Med 1984; 10: 1-15. 
${ }^{7}$ Hutson JM. A biphasic model for hormonal control of testicular descent. Lancet 1985; ii: 419-21.

${ }^{8}$ Davies TW, Williams DRR, Whitaker RH. Risk factors for undescended testis. Int J Epidemiol 1986; 15: 197-201.

${ }^{9}$ Swerdlow AJ, Wood KH, Smith PG. A case-control study of cryptorchidism. J Epidemiol Community Health 1983; 37: $238-44$

${ }^{10}$ International Statistical Classification of Diseases, Injuries and Causes of Death; 1965 revision adapted for indexing of hospital records and morbidity statistics (in Swedish). Stockholm, 1968.

${ }^{11}$ Miettinen OS. Simple interval estimation of risk ratio. $\mathrm{Am}$ $J$ Epidemiol 1974; 100: 515-6.

12 Edwards JH. The recognition and estimation of cyclic trends. Ann Hum Genet 1961; 25: 83-7.

${ }^{13}$ Czeizel A. Aetiological studies of isolated common congenital abnormalities in Hungary. Budapest: Akademiai Kiado, 1984.

14 Jackson MB, Swerdlow AJ. Seasonal variations in cryptorchidism. J Epidemiol Community Health 1986; 40: 210-3.

${ }^{15}$ Knox EG, Cummins C. Birth dates of men with cancer of the testis. J Epidemiol Community Health 1985; 39: 237-43.

${ }^{16}$ Källén B, Bertollini B, Castilla E, et al. A joint international study on the epidemiology of hypospadias. Acta Paediatr Scand (Suppl) 1986; 324.

${ }^{17}$ Källén B. Epidemiology of human reproduction. Boca Raton, Florida: CRC Press, 1988; 177-8.

${ }^{18}$ Kauppila A, Kivelä A, Pakarinen A, Vakkuri O. Inverse relationship between melatonin and ovarian activity in a region with a strong seasonal contrast in luminosity. $J$ Clin Endocrinol Metab 1987; 65: 823-8.

19 Jarrige JF, Tlemcani O, Boucher D. Gonadal function in male offspring of pinealectomized female rats. Acta Endocrinol 1987; 116: 247-52.
${ }^{20}$ Depue RH. Maternal and gestational factors affecting the risk of cryptorchidism and inguinal hernia. Int $J$ Epidemiol 1984; 13: 311-7.

${ }^{21}$ Beard M, Melton LJ, O'Fallon WM, Noller KL, Benson RC. Cryptorchidism and maternal estrogen exposure. Am J Epidemiol 1984; 120: 707-16.

22 Hadziselimovic F. Cryptorchidism. Management and implications. Berlin: Springerverlag, 1983.

${ }^{23}$ Källén B. Hyperemesis during pregnancy and delivery outcome: a registry study. Eur J Obstet Gynecol Reprod Biol 1987; 26: 291-302.

${ }^{24}$ Swerdlow AJ, Huttly SRA, Smith PG. Prenatal and familial associations of testicular cancer. $\mathrm{Br} J$ Cancer 1987; 55: 571-7.

${ }^{25}$ Svensson J. Male hypospadias. 625 cases, associated malformations and possible aetiological factors. Acta Pediatr Scand 1979; 68: 587-92.

${ }^{26}$ Andren L. Pelvic instability in newborns with special reference to congenital dislocation of the hip and hormonal factors. Acta Radiol (Suppl) (Stockh) 1962; 212.

${ }^{27}$ Burton MH, Davies TW, Raggatt PR. Undescended testis and hormone levels in early pregnancy. $J$ Epidemiol Community Health 1987; 41: 127-9.

${ }^{28}$ Barbieri RL, Saltzman D, Phillippe M, et al. Elevated $\beta$-human chorionic gonadotropin and testosterone in cord serum of male infants of diabetic mothers. $J$ Clin Endocrinol Metabol 1985; 61: 976-9.

${ }^{29}$ Heikinheimo M, Seppala M. Amniotic fluid human chorionic gonadotropin in late pregnancy; elevated levels in toxemia with intrauterine growth retardation. $\mathrm{Am} \mathrm{J}$ Obstet Gynecol 1983; 146: 766-75.

Accepted for publication April 1989 